\title{
Use of Silicon Microstrip Detectors for Precise Measurement of High Momenta
}

\section{WA76 Collaboration}

\begin{abstract}
T.A. Armstrong 1,*), M. Benayoun 6), W. Beusch 1), I.J. Bloodworth 2), M. Caponero 3), J.N. Carney 2), R. Childs 2), B.R. French 1), B. Ghidini 3), Y. Goldschmidt-Clemont 1,7),
\end{abstract} A. Jacholkowski 1), J. Kahane 6), J.B. Kinson 2), A. Kirk 2), V. Lenti 3), Ph. Leruste 6), A. Malamant 6), J.L. Narjoux 6), F. Navach 3), A. Palano 3), E. Quercigh 1), N. Redaelli 1,**), L. Rossi $1{ }^{* * *}$ ), M. Sené 6), R. Sené 6), H.R. Shaylor 2), M. Stassinaki 4), M.T. Trainor 2), G. Vassiliadis 4), O. Villalobos Baillie 2), M.F. Votruba 2), G. Zito 3) and R. Zitoun 5)

\begin{abstract}
The WA76 experiment performed at the $\Omega$ facility at CERN required a high precision measurement of the forward produced particle whose momentum was close to $300 \mathrm{Gev} / \mathrm{c}$. A telescope made of 12 silicon microstrip detectors has been built and operated for such a purpose.
\end{abstract}

\section{Submitted to Nuclear Instruments and Methods}

\footnotetext{
1) CERN, CH-1211 Geneva 23, Switzerland

2) University of Birmingham, Physics Department,Birmingham,U.K.

3) Dip. di Fisica dell'Universita and Sez. INFN, Bari, Italy

4) Athens University, Physics Department, Athens, Greece

5) LPNHE, Universitès de Paris VI et VII, Paris, France

6) Collège de France, Paris, France

7) Deceased

*) Present address: Pennsylvania State University, University Park, USA

**) Present address: INFN and Dip. di Fisica, Milan, Italy

***) Present address: INFN and Dip. di Fisica, Genoa, Italy
} 


\section{Introduction}

The aim of the WA76 experiment [1] is to study the reaction

$$
\mathrm{p} \mathrm{p} \rightarrow \mathrm{p}_{\mathrm{f}}\left(\mathrm{X}^{0}\right) \mathrm{p}_{\mathrm{s}}
$$

at $300 \mathrm{GeV} / \mathrm{c}$ incident momenturn where the subscripts $\mathrm{f}$ and $\mathrm{s}$ indicate, respectively, the fastest and slowest particles in the laboratory system and $\mathrm{X}^{0}$ represents the central system decaying into charged and neutral final states. Reaction (1) was isolated through:

1. the selection of a fast and a slow particle (this is done by the trigger and briefly illustrated in chapter 2);

2. the requirement of transverse and longitudinal momentum balance (between the incident hadron and the outgoing particles).

In order to get the momentum balance with enough accuracy to have a negligible background from events with undetected siow $\pi^{0}$ s we used the standard configuration of the $\Omega$ spectrometer for the low momentum tracks, but we increased the spatial accuracy in the small solid angle where the fast particle was measured. The required accuracy on the trajectory was obtained by using microstrip detectors [2]. This technique is particularly well suited to perform this measurement since the fast tracks pass very close $(\approx 1 \mathrm{~cm})$ to the beam tracks and detectors of relatively small active area $\left(\approx 10 \mathrm{~cm}^{2}\right)$, like silicon microstrips, may therefore cover the desired acceptance. Moreover the high beam intensity $(2 \mathrm{MHz}$ during the spill), needed to get the required sensitivity, ruled out alternative detectors with similar accuracy. For example high pressure drift chambers could not be used because of their long memory time and the distortions induced by space charge effects.

A telescope, made of silicon microstrip detectors, already built and operated for the experiment IMPACT [3] at the $\Omega$ spectrometer, has been modified and improved with the addition of new detectors so that it could fulfill the stringent requirements put forward by the WA76 experiment. The aim of this paper is to describe the microscrip telescope and its role in the selection of the reaction (1).

This paper is organized as follows : in section 2 we describe the apparatus and the trigger, and in section 3 the fast track reconstruction method. In section 4 we discuss how we aligned and controlled the microstrip detectors and finally we draw our conclusions in section 5 . 


\section{Experimental layout and trigger}

The layout of the $\Omega$ spectrometer used in the WA76 experiment is shown in fig. 1 and comprises:

1. A set of detectors to perform an accurate measurement of the incident beam direction. This was achieved by using four scintillator hodoscopes ( $2 y$ and $2 z$ planes) having $1 \mathrm{~mm}$ width and partially overlapped to produce $1 / 3 \mathrm{~mm}$ logical slabs; four $50 \mu$ pitch $\mu$-strip detectors (2y and $2 z$ planes); four MWPC's ( $2 y$ and $2 z$ planes) with $1 \mathrm{~mm}$ spacing used essentially to backup the hodoscopes and $\mu$-strips system in case of inefficiency. The beam momentum was $300 \mathrm{GeV} / \mathrm{c}$ with a momentum bite of $0.25 \%$.

2. A $60 \mathrm{~cm}$ long hydrogen target and a system of scintillator hodoscopes for the trigger definition (as described below).

3. The standard $\Omega$ set - up which consists of 16 proportional chambers (A and B) and two large drift chambers (DC). This layout was used to measure the medium momentum tracks leaving the interaction region. In addition 16 planes of MWPC (C) were used to measure the slow proton trajectory.

4. A system of $8 \mu$-strips detectors (see fig. 2) placed downstream of the two drift chambers in two sets of 4 planes placed at $\approx 6 \mathrm{~m}$ and $\approx 10 \mathrm{~m}$ from the $\Omega$ centre in order to measure the fast track direction. The 6 metre planes $(2.5 \times 2.5 \mathrm{~cm})$ had a sequence $(y, z, y, v)$ and the 10 metre planes $(5 \times 5 \mathrm{~cm})$ a sequence $(\mathrm{u}, \mathrm{z}, \mathrm{y}, \mathrm{y})$ where the $\mathrm{v}$ and $\mathrm{u}$ planes had inclined strips at -10.12 and +10.12 degrees with respect to the vertical. The first y plane at 6 meters was larger $(5 \times 5$ $\mathrm{cm}$ ) than the others and the last y plane at $10 \mathrm{~m}$ was shifted by $1 \mathrm{~cm}$ with respect to the other planes so they both intersect the beam line for calibration purposes. A small MWPC (one $z$ plane, $1 \mathrm{~mm}$ spacing) (TOM) was placed at $6 \mathrm{~m}$ to backup inefficiencies in the pattern recognition.

5. A forward identification system consisting of scintillator hodoscopes ( $\mathrm{H} 1, \mathrm{H} 2$ and $\mathrm{H} 3$ ) and Cherenkov counters $(\mathrm{Cl}$ and $\mathrm{C} 2)$ for charged particle identification, and a large electromagnetic calorimeter [4] for the measurement of $\gamma^{\prime} \mathrm{s}$. 
The small $\mu$-strip detectors were provided by Enertec-Schlumberger (France), while the large $\mu-$ strip detectors were provided by Micron Semiconductor (UK). All the detectors have a pitch of 50 $\mu \mathrm{m}$ and a thickness varying from 300 to $450 \mu \mathrm{m}$. Each strip is read out individually; after a hybrid charge preamplifier (MSD2 [5]) that shapes the pulse to a Gaussian with a width of 50 nsec FWHM, each signal above a selectable threshold is stored into the same encoding system used for the read out of the $\Omega$ proportional chambers [6]. The data are finally read out through Romulus interfaces into a VAX 780 computer. The trigger of the experiment required:

1. A fast particle crossing the downstream $\mu$-strip telescope. This requirement was fulfilled by asking for a coincidence between two scintillation counters (called $A_{1}$ and $A_{2}$ ) placed close to each set of downstream $\mu$-strip detectors and designed to cover their acceptance.

2. A slow particle defined by demanding one hit on any of the fourteen horizontal slabs of the Slow Proton Counter (see fig. 1), $\left(56 \times 88 \mathrm{~cm}^{2}\right)$, and $\geq 1$ hit on a single plane of the MWPCs situated on one side of the target.

3. In order to reduce the backward diffraction or excitation we requested no hit in the other three sides of the box TS counter which was left open at its front end to allow particles produced centrally to escape downstream.

4. To reduce the forward diffraction or excitation we requested no hit in two counters (DFC) of dimensions $\left(32 \times 30 \mathrm{~cm}^{2}\right)$ which were placed on either side of the beam and just downstream of Cherenkov $\mathrm{C} 1$.

The forward $\mu$-strips and scintillation counters at 6 and 10 metres were placed (see fig. 2) in such a way that beam particles were not crossing the $\mu$-strips but passed as close as possible to them. The beam was focused downstream at $\approx 8 \mathrm{~m}$ from the $\Omega$ centre giving a beam profile as narrow as possible in the $y$ direction $(\sigma(y)=1.5 \mathrm{~mm}, \sigma(z)=2.5 \mathrm{~mm})$. The beam, however, crossed the two extended planes, mentioned above, so as to have a constant monitor of the $\mu$-strip positions. Beam tracks were, for this purpose, recorded periodically during the data taking. The raw hit distribution on the $6 \mathrm{~m}$ 
extended plane is shown in fig. 3 where the beam profile can be seen on the right side of the sharp edge due to the $A_{1}$ trigger counter. This layout, with the fast track accepted on the same side of the slow proton automatically excluded elastic scatters.

The data taking has been performed in two periods of 17 days; the beam flux was held at $4 \times 10^{6}$ particles on a $4 \times 7 \mathrm{~mm}^{2}$ (fwhm) spot over a spill of $2.8 \mathrm{sec}$. The beam intensity was mostly limited by the capability of the $\mu$-strip detectors to handle high intensity fluxes over periods of several weeks without a major increase in reverse current.

\section{Events reconstruction}

The event reconstruction has been performed by using a version of the pattern recognition and geometrical reconstruction program TRIDENT [7] modified for the purposes of the present experiment.

The beam reconstruction has been performed by finding straight lines in the $(x, z)$ and $(x, y)$ projections after subtracting the bending due to the magnetic field. The overall reconstruction efficiency for finding the beam track in both projections was $98 \%$.

The fast track pattern recognition has been performed by using the eight $\mu-$ strip forward planes with the help of the TOM chamber. The algorithm involve iterating several times along the $(x, y)$ and $(\mathrm{x}, z)$ projections in order to use the information from the inclined strips. The resulting efficiency for finding the track in both projections was $\approx 96 \%$.

Typical events are shown in fig. $4 a$ (six outgoing tracks) and $4 b$ (two charged and neutrals). The fast particle crosses a region of the MWPC's and DC's close to the beam trajectory where these detectors are very inefficient and no information is thus available for the reconstruction of the fast particle trajectory. The fast track momentum has then been computed by using the spline fit method [8] with three points only, i.e. the main vertex and the intersections with the $6 \mathrm{~m}$ and $10 \mathrm{~m}$ planes of the straight line reconstructed with the forward $\mu-$ strips.

The main vertex was found by the reconstruction program as a weighted mean of all the outgoing tracks and the incoming beam. This vertex point has been readjusted by taking as $\mathrm{x}$ coordinate the one 
found by TRIDENT but taking the more precise $y$ and $z$ coordinates extrapolated from the beam. This point has been then used, in addition to all the measured points, to recompute the track momentum. As a result of this recomputation we have observed a clear narrowing of the missing transverse and longitudinal momentum distributions indicating an improvement of the experimental resolution.

\section{Alignment and calibration}

The alignment of the whole system, critical for the isolation of momentum balancing events, has been performed by using beam tracks which have been steered to cross the full $\mu$-strip system. Starting from an accurate geometrical measurement of the positions of the MWPC's and drift chambers an iterative procedure has been performed which computed the difference between the measured position of each device with respect to the traced beam position. Each device was alternatively included (with the proper weight) and excluded from the momentum determination of the beam.

The result of the alignment procedure is summarized in fig. 5 where we have plotted the resulting beam momentum distribution. The distribution is well centred at $300 \mathrm{GeV} / \mathrm{c}$ and has $\sigma=2.7 \mathrm{GeV} / \mathrm{c}$, in agreement with the value predicted by a Monte-Carlo simulation of the experimental set-up.

The spatial accuracy of the $\mu$-strip detectors has been determined as follows: the reconstructed line in the forward $\mu$-strip detectors has been compared with the coordinate calculated from the information coming from the individual planes. The resolution function, determined with the method described in ref. [9] was found to have a $\sigma=14.8 \mu \mathrm{m}$ which is close to that expected $(\sigma=50 / \sqrt{ } 12=14.4 \mu \mathrm{m})$. An example of the distribution of the difference of the actual hit position from that of the predicted hit position is shown in fig. 6 for a specific $\mu$-strip. This distribution has $\sigma=19.5 \mu \mathrm{m}$ in agreement to that expected for a $\mu$-strip placed in this position.

The physics of this experiment is sensitive to a transverse displacement of the $\mu-$ strip detectors of the order of 10 microns, and it was therefore necessary to monitor the detector positions continuously. For this purpose, the recorded beam tracks were traced, starting from the reconstructed beam direction in the beam layout, up to the $6 \mathrm{~m}$ and $10 \mathrm{~m}$ planes. The results of this monitoring were kept as a data bank for the events reconstruction. 
We have selected a sample of events having four reconstructed outgoing particles and which balanced the electric charge. For these events we have plotted in fig. 7a the missing transverse momentum between the incident beam and the outgoing particles. The distribution shows a narrow peak at threshold indicating the presence of events which balance the transverse momentum. By requiring $\mid$ missing $\mathrm{Pz} \mid<0.08 \mathrm{GeV} / \mathrm{c}$ and $\mid$ missing $\mathrm{Py} \mid<0.16 \mathrm{GeV} / \mathrm{c}$ we have plotted in fig. $7 \mathrm{~b}$ the missing $\mathrm{Px}$ distribution which shows the expected signal, on negligible background, for events which balance the longitudinal momentum. The distribution has $\sigma=6.5 \mathrm{GeV} / \mathrm{c}$ and the mean value and the standard deviation of this distribution as well as the presence of a clear peak in the missing transverse momentum distribution have been taken as the parameters used to check the quality of the reconstructed data. The width of the missing longitudinal momentum distribution is mostly due to the error on the momentum of the fast track. The increase of this error, with respect to the momentum determination performed on beam tracks is entirely due to the extrapolation of the measured beam direction from the last measured point up to the vertex of the event.

The test on data quality has been performed for each tape. A calibration run, using beam tracks, has been used to obtain new alignment values for data which deviated from the standard quality. Fig. 8 shows the $\sigma$ variation as a function of time for the second period of data taking. The value of $\sigma$ shows a slight increase with time which could be due to the progessive deterioration in the performances of the $\mu$-strip detectors. From the missing transverse and longitudinal momentum distributions shown in fig. 7 it can be seen that we have achieved our aim of using $\mu$-strip detectors to measure the fast track sufficiently accurately to to perform exclusive physics at $300 \mathrm{GeV} / \mathrm{c}$ incident momentum. In order to study the possibility of identifying final states involving kaons or protons, we have plotted in fig. $9 \mathrm{a}$ the Ehrlich mass [10] for 4-prong events which balance momentum. The peak at the squared $\pi$ mass is the signal of the centrally produced $\pi^{+} \pi^{-}$final state, and a shoulder can be seen at the squared kaon mass. We have then required one of the central particles to be identified as a $\mathrm{K}, \mathrm{p}$ or ambiguous $\mathrm{K} / \mathrm{p}$ and the second, if reaching the Cherenkov system, to have a mass identification compatible with being a $\mathrm{K}$ or $\mathrm{p}$. This selection results in the distribution of fig. $9 \mathrm{~b}$ where three structures can be seen: a peak at the squared $\pi$ mass which is the indication of a contamination from the $\pi^{+} \pi^{-}$reaction where one of the two pions has been misidentified as a $\mathrm{K}$ or $\mathrm{p}$ from the Cherenkov information, and two peaks 
centred at the squared $\mathrm{K}$ and $\mathrm{p}$ masses which are the signals of the $\mathrm{K}^{+} \mathrm{K}^{-}$and $\mathrm{p} \overline{\mathrm{p}}$ final states respectively. This plot shows that the experimental resolution at this energy is sufficient to separate the $\mathrm{K}^{+} \mathrm{K}^{-}$from the $\pi^{+} \pi^{-}$channel.

\section{Conclusions}

We have used $\mu$-strip silicon detectors in order to measure the momentum and the direction of fast $(\approx 300 \mathrm{Gev} / \mathrm{c})$ particles. The spatial accuracy and the stability of these detectors have allowed us to isolate, for the first time, exclusive hadronic reactions in the central rapidity region at $300 \mathrm{GeV} / \mathrm{c}$ incident momentum. 


\section{Acknowledgments}

We would like to thank the technical staff who made this experiment possible by helping in the preparation, data taking and offline analysis. We acknowledge the EF detector group (in particular E.H.M. Heijne, P. Jarron and A. Menetrey), and the $\Omega$ support group (in particular R. Pegaitaz). We thank E. Barbarito (Bari) for his help in the test of the elecronics associated with the $\mu-$ strip detectors, M. Sitruk (CDF) for the help during the data taking and A. Loconsole (Bari) for having processed part of the data through the reconstruction programs. 


\section{References}

[1] CERN Proposals SPSC/82-3/P175 and SPSC/84-62/P204

[2] E.H.M. Heijne et al., Nucl. Instr. and Meth. 178 (1980) 331;

B. Hyams et al., Nucl. Instr. and Meth. 205 (1983) 99.

[3] J.F. Baland et al., Nucl. Phys. 1B (1988) 303.

[4] L. Bachman et al., Nucl. Instr. and Meth. 206 (1983) 85.

[5] A. Beer et al., 'A pulse width modulation circuit for the remote readout of very long shift registers', Yellow report CERN $78-14$ (1978).

[6] P. Jarron and M. Goyot, Nucl. Instr. and Meth. 226 (1984) 156.

[7] J.C. Lassalle et al., Nucl. Instr. and Meth. 176 (1980) 371.

[8] H. Wind, Nucl. Instr. and Meth. 115 (1974) 431.

[9] M. Benayoun et al., Application de l'analyse multidimensionelle a l'etude d'un spectrometre focalisant. LPC 80-9, College the France, Paris.

[10] R. Ehrlich et al., Phys. Rev. Lett. 20 (1968) 686. 


\section{Figure captions}

Fig. 1 Layout of the CERN $\Omega$ spectrometer as used for the WA76 experiment.

Fig. 2 Layout of the $\mu$-strips and scintillators used to measure the beam and the fast particle.

Fig. 3 Raw hits distribution on the extended $\mu-$ strip at $\approx 6 \mathrm{~m}$ from the $\Omega$ centre. The beam shape, allowed for calibrations, is visible on the right side. The sharp edge is due to the $\mathrm{A}_{1}$ counter trigger.

Fig. 4 Typical events. The beam $\mu$-strips, the MWPC's, the drift chambers and the forward $\mu$-strips are shown from left to right. The dashed line indicates the reconstructed track in the forward $\mu$-strips. a) Six outgoing charged tracks event; b) two charged and neutrals.

Fig. 5 Beam momentum after the alignment of the whole system. The curve represents a Gaussian fit to the data.

Fig. 6 Distribution of residuals on a forward $\mu-$ strip detector plane. The curve is a Gaussian fit.

Fig. 7 a) Missing transverse momentum distribution for events with four outgoing tracks;

b) Missing longitudinal momentum after $P_{t}$ cut.

Fig. $8 \quad$ b) Variation of the $\sigma$ of the missing longitudinal momentum distribution for 4 prongs events as function of time.

Fig. 9 a) Ehrlich mass distribution for all the four prong events;

b) Ehrlich mass distribution after having used the Cherenkov information. 


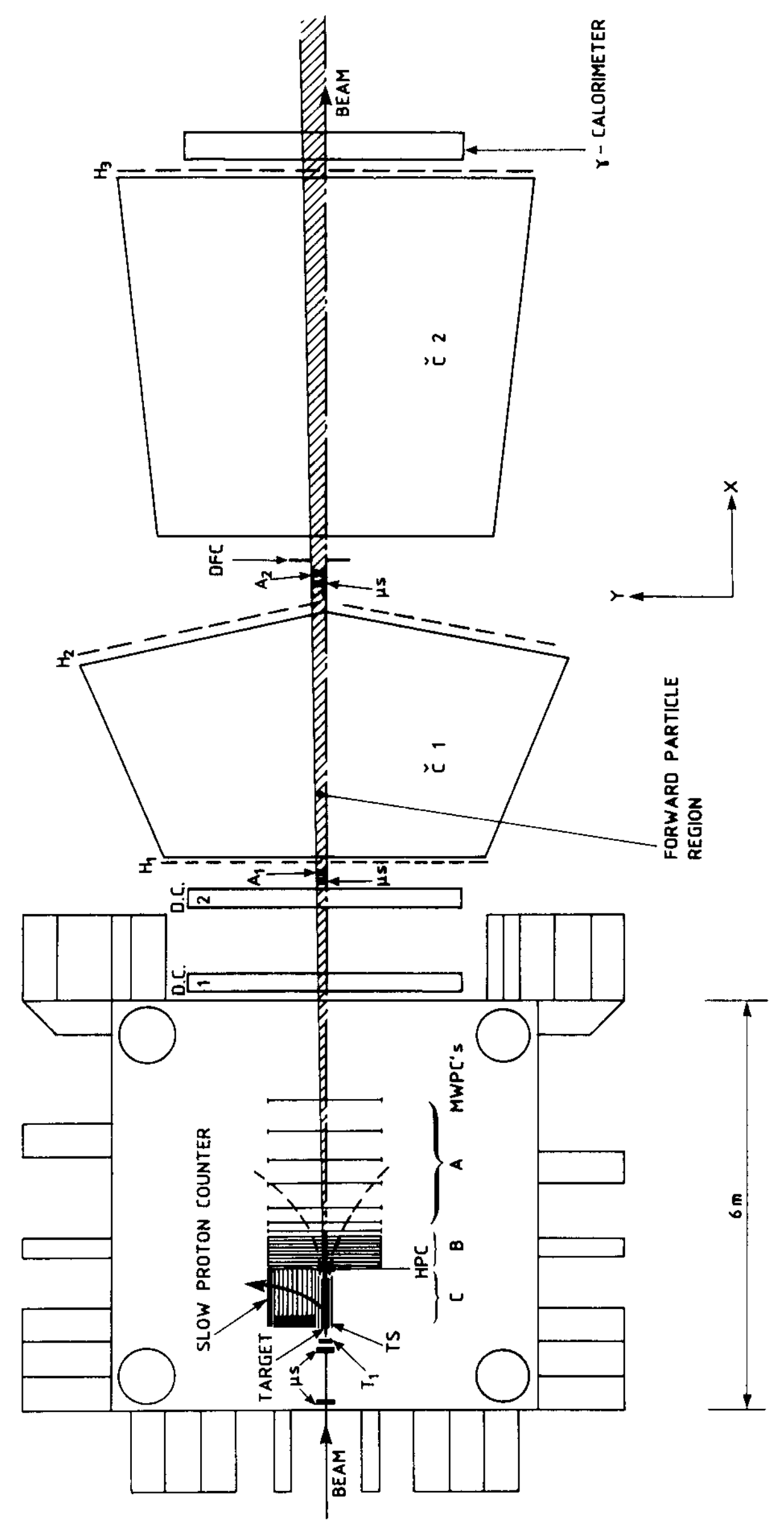

분 


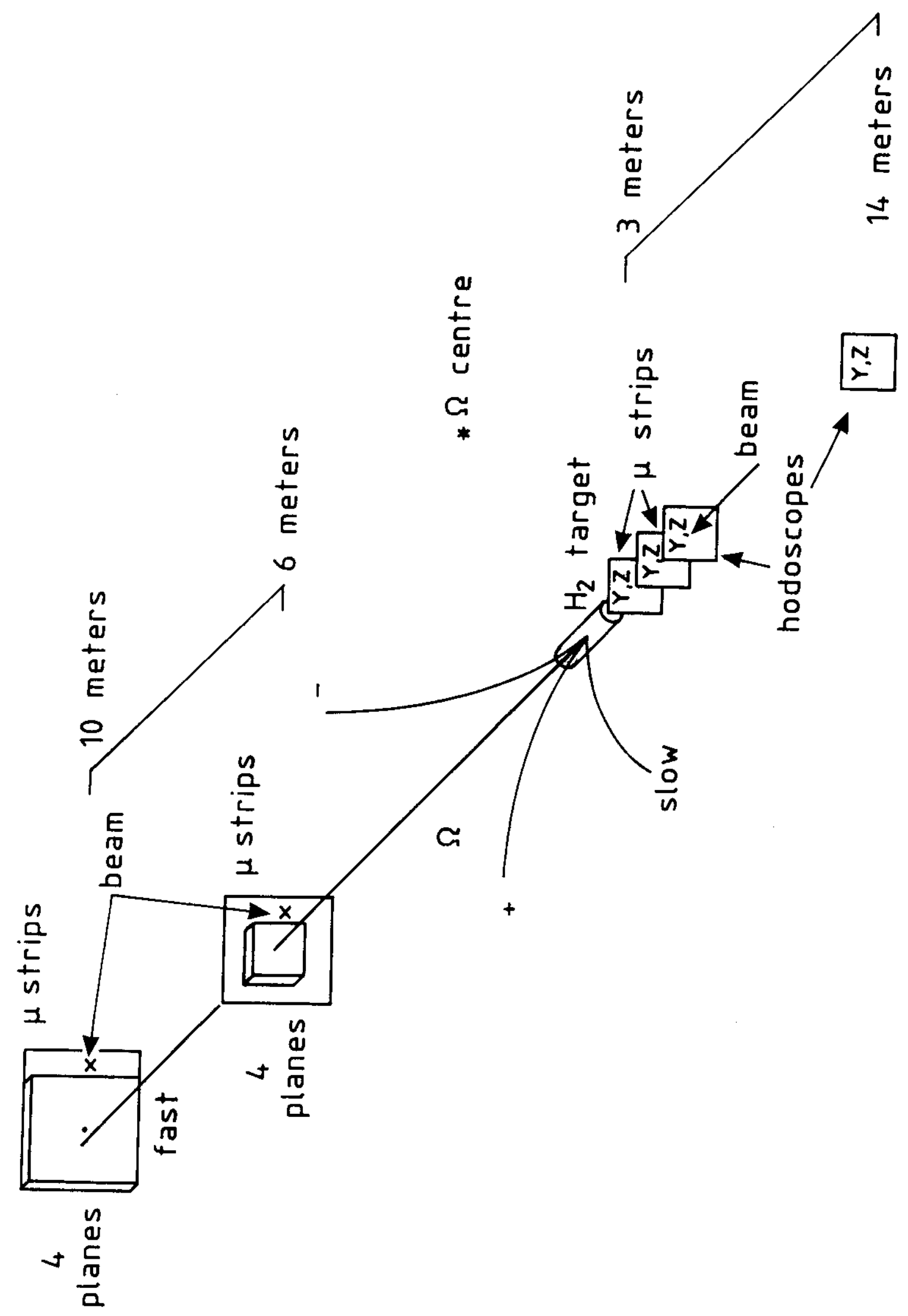




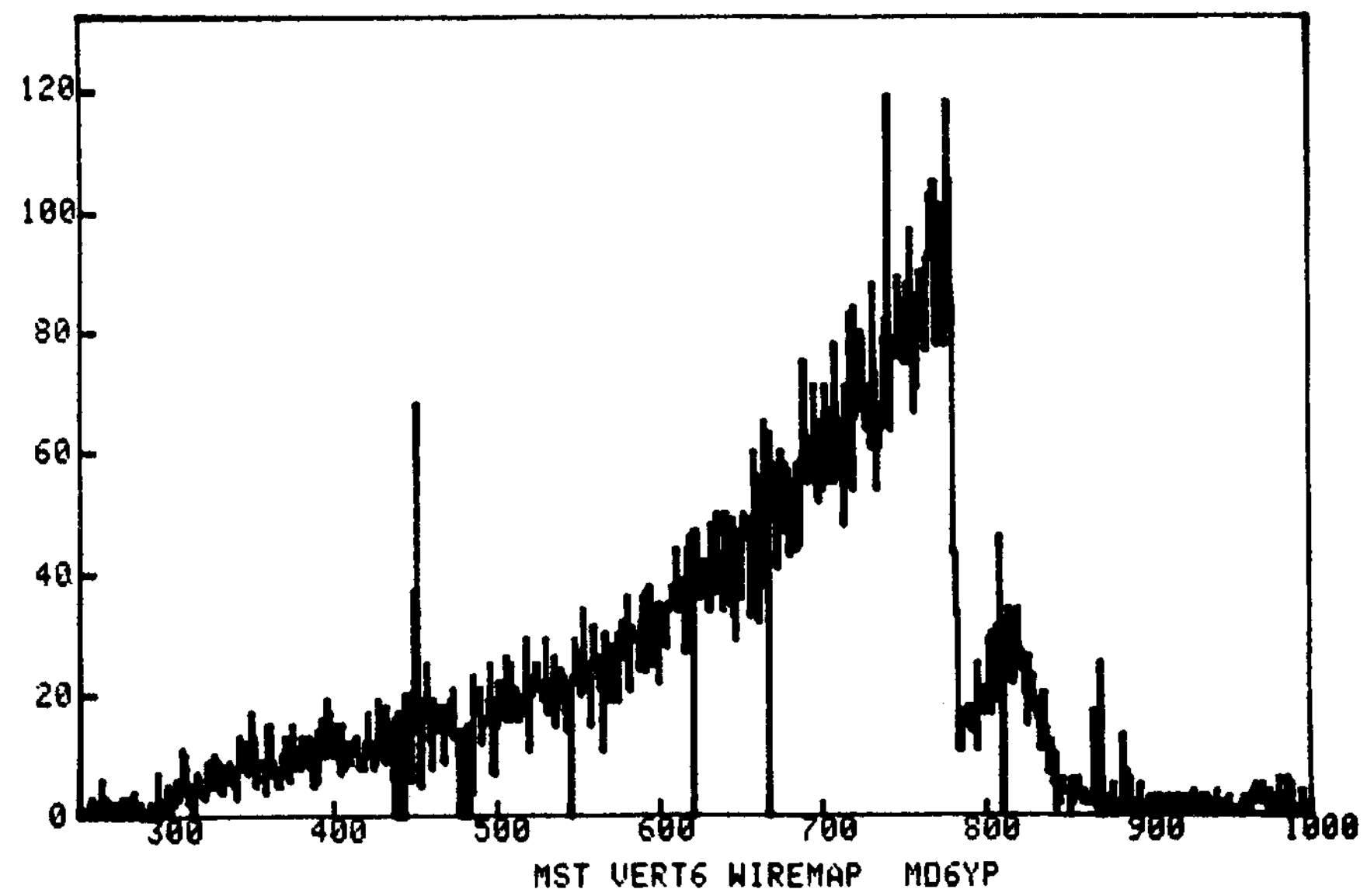

Fig. 3 

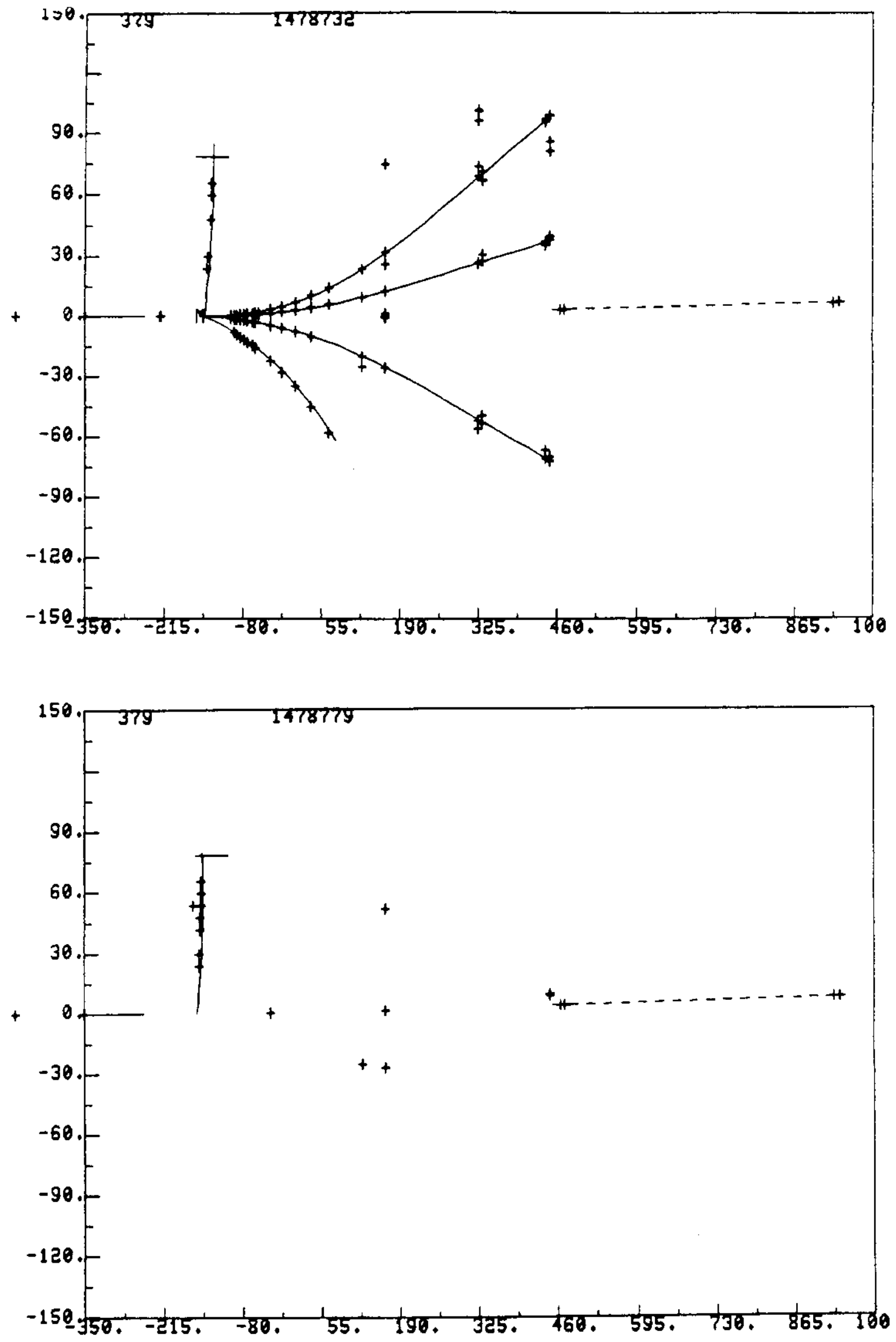

Fig. 4 


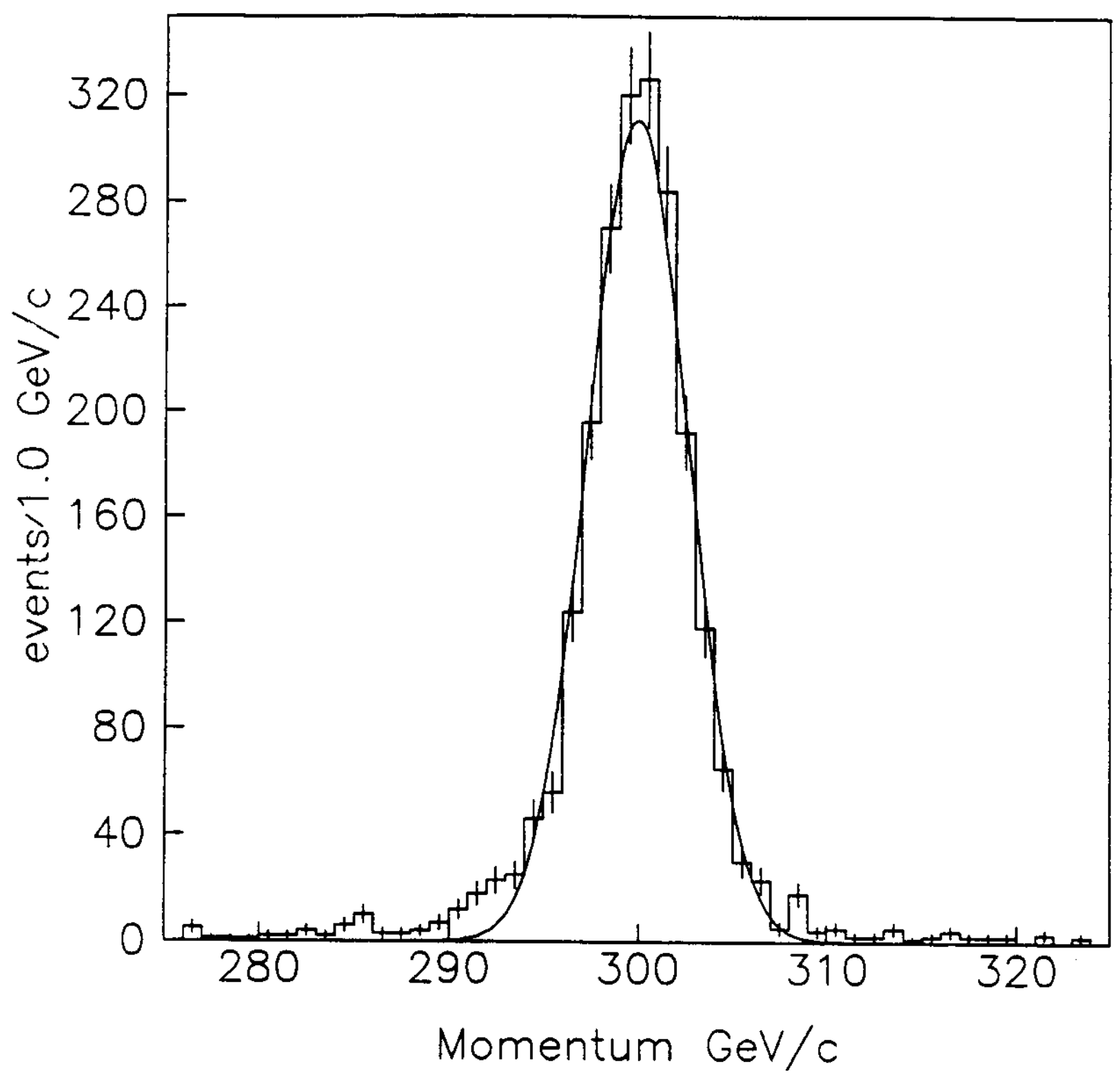

Fig. 5 


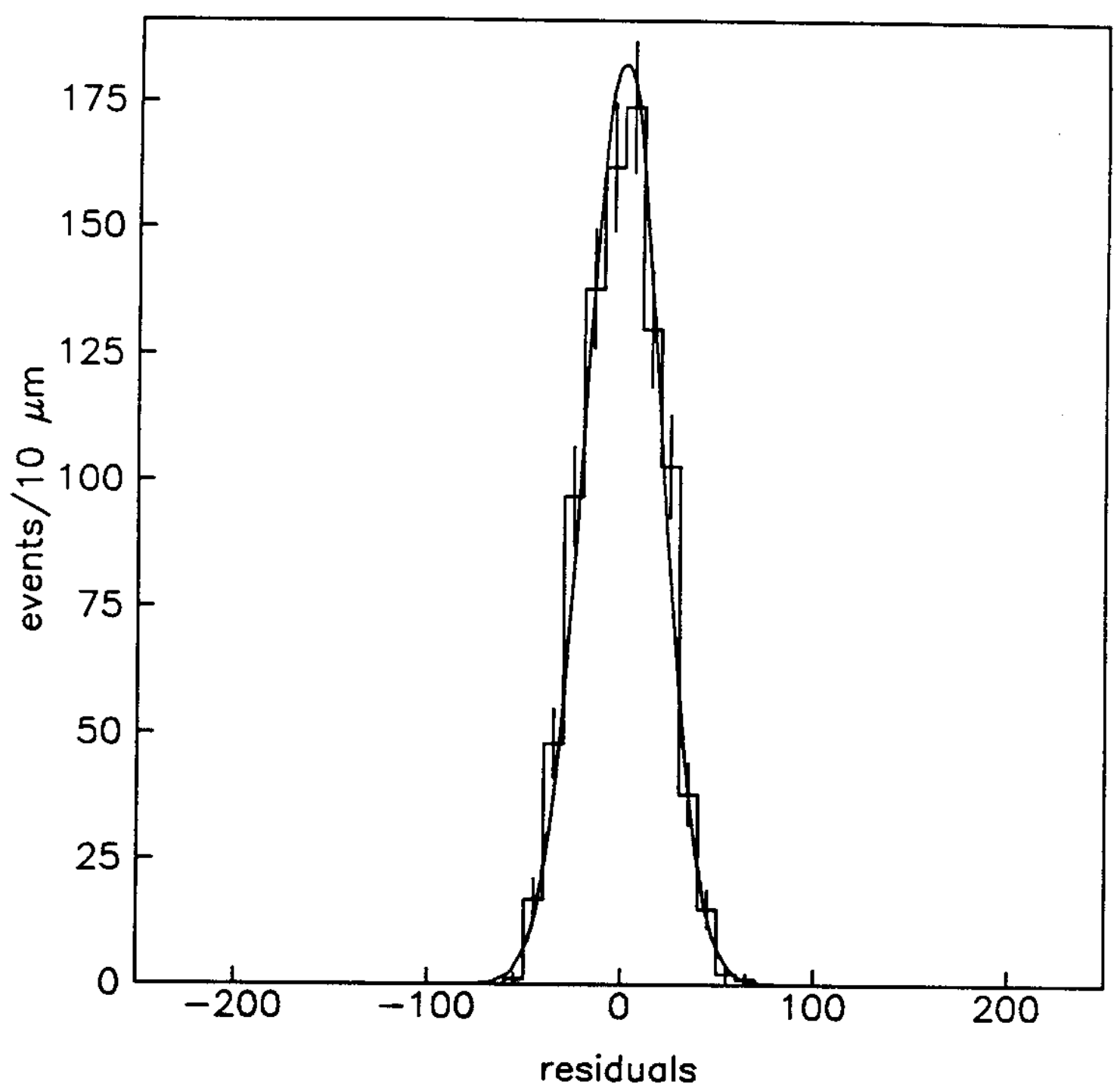

Fig. 6 

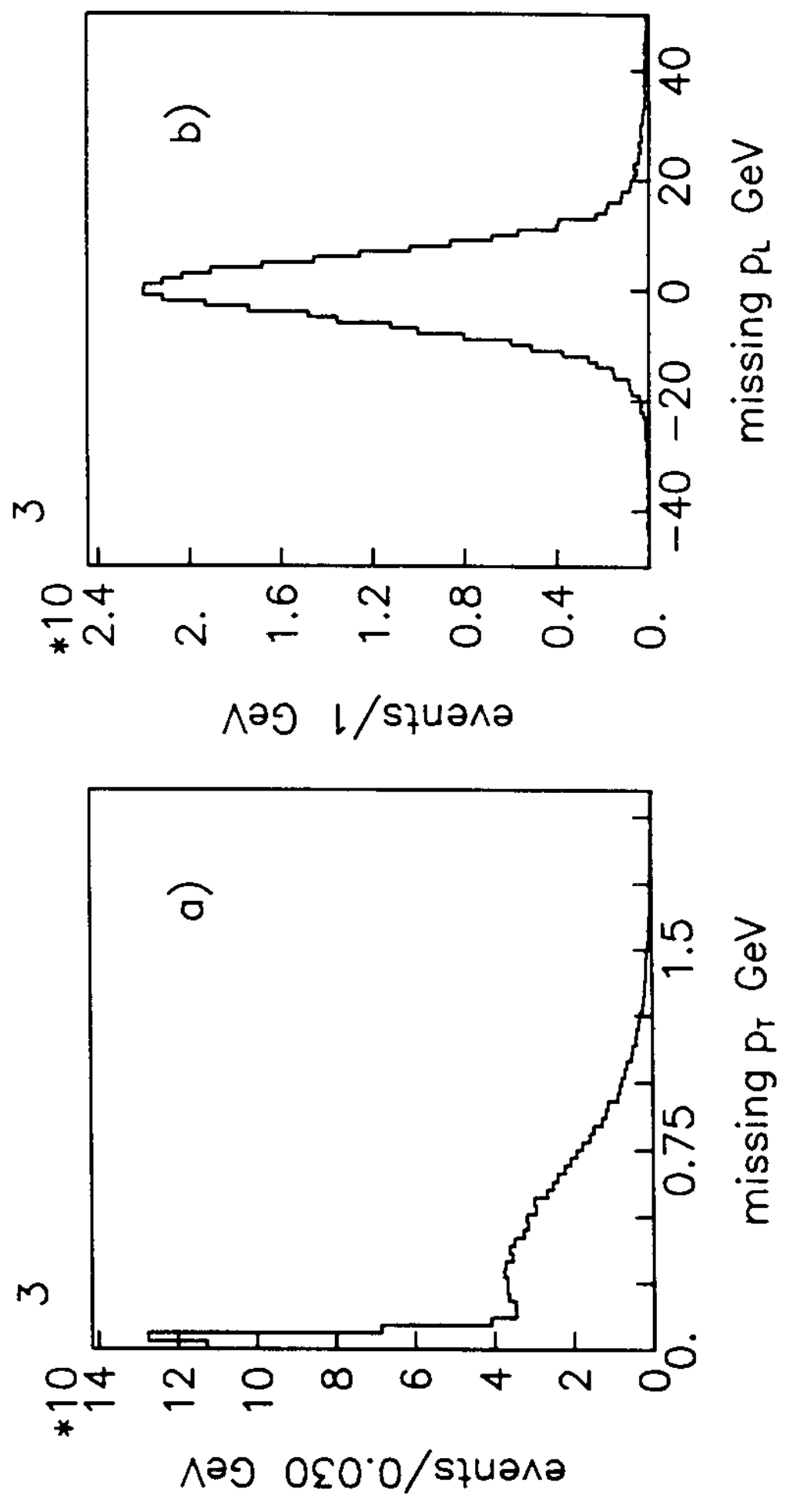

$r$
$\dot{s}$
$\dot{n}$
$\dot{n}=1$ 


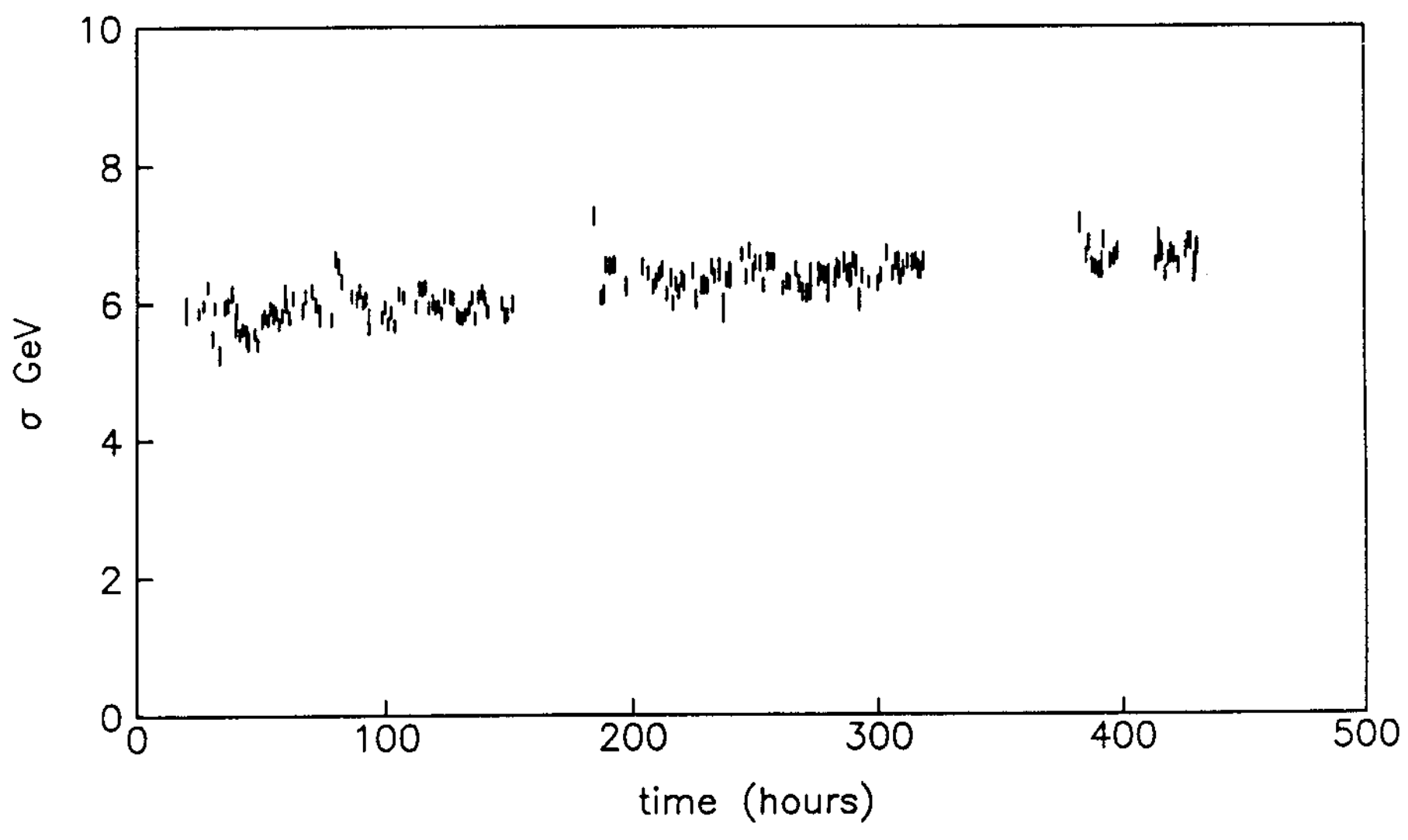

Fig. 8 


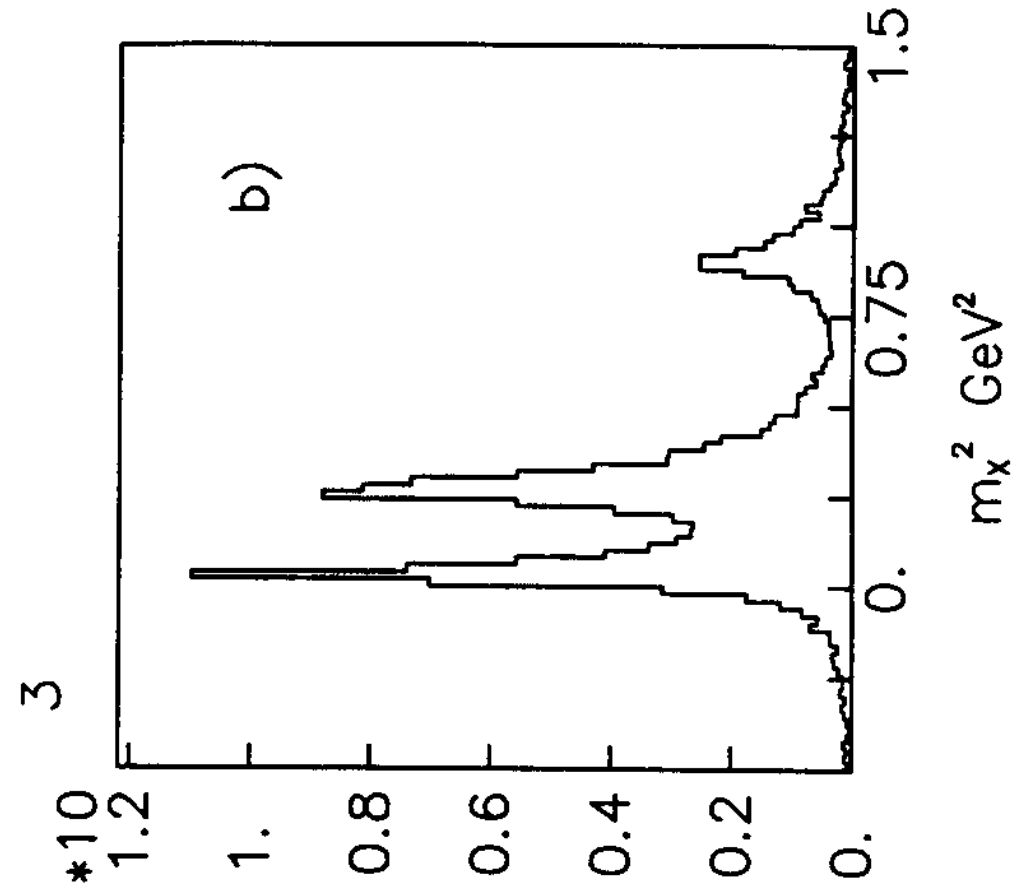

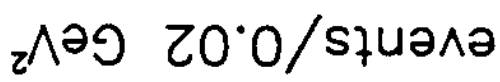

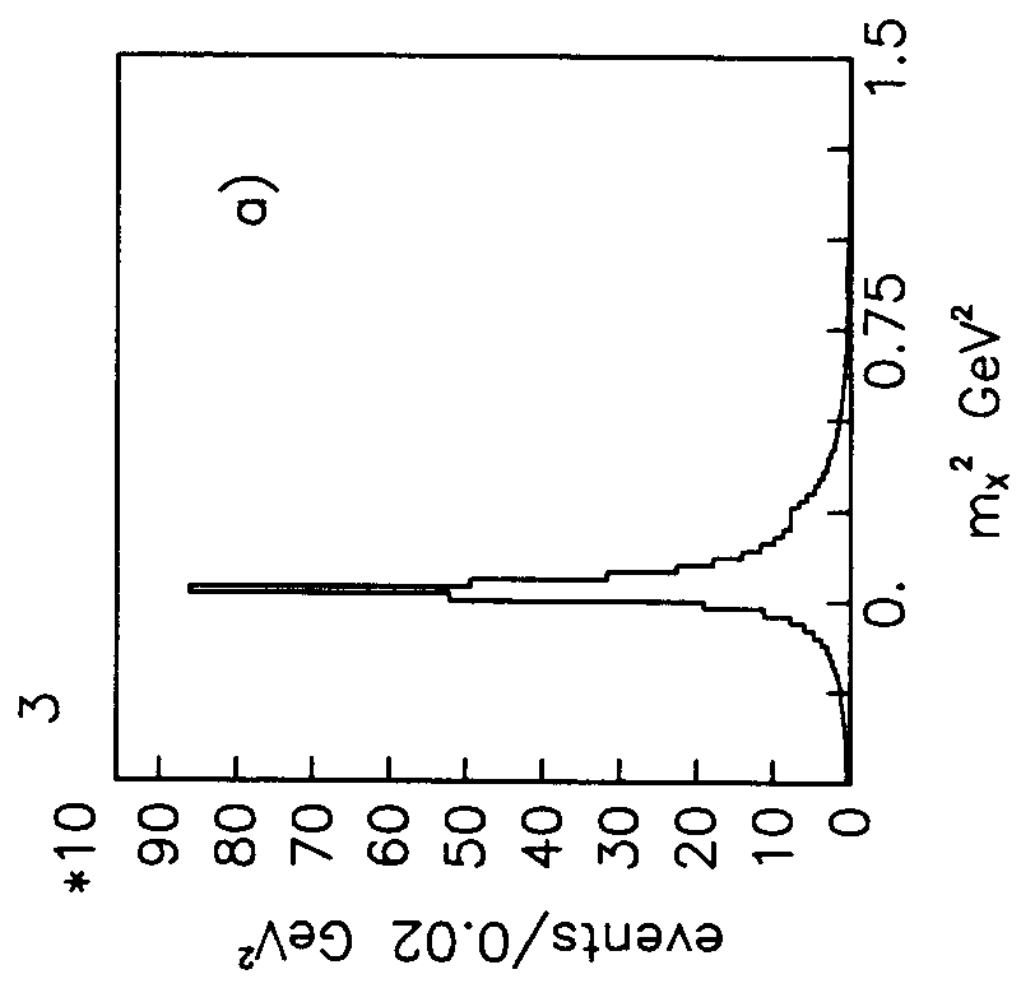

$a$
0
0
$0=1$ 